\section{ECOLOGÍA HUMANA. NUEVOS DESAFÍOS PARA LA ECOLOGÍA Y LA FILOSOFÍA}

\author{
Luca Valera \\ Pontificia Universidad Católica de Chile \\ ORCID: https://orcid.org/0000-0002-1693-396X \\ luvalera@uc.cl
}

Cómo citar este artículo/Citation: Valera, L. (2019). Ecología humana. Nuevos desafíos para la ecología y la filosofía. Arbor, 195 (792): a509. https://doi.org/10.3989/arbor.2019.792n2010

Recibido: 30 septiembre 2015. Aceptado: 28 abril 2016.

RESUMEN: El desarrollo actual de la ecología ha abierto nuevos desafíos para la reflexión filosófica contemporánea. En este sentido, ya no es posible interpretar la naturaleza humana a partir de una reflexión sobre el hombre como un ser aislado de su ambiente. El paradigma de ecología humana que se presenta aquí quiere heredar la reflexión sobre la filosofía de la naturaleza (y también sobre la filosofía de la naturaleza humana), proponiendo la cuestión ecológica actual como un tema eminentemente antropológico: el lugar del hombre en el cosmos indica asimismo su esencia. Esta tarea nos obliga a replantear la especificidad del ser humano en relación con el mundo a través del tema del habitar, que nos invita a reflexionar sobre las actividades del custodiar y del construir la casa. Se hace así posible replantear el tema ecológico actual como una crisis predominantemente antropológica: para sanar la herida del ecosistema, primero hay que curar la fractura dentro del hombre.

PALABRAS CLAVE: Ecología humana; naturaleza humana; ética ambiental; habitar; casa.

\section{HUMAN ECOLOGY. NEW CHALLENGES FOR ECOLOGY AND PHILOSOPHY}

Copyright: (C) 2019 CSIC. Este es un artículo de acceso abierto distribuido bajo los términos de la licencia de uso y distribución Creative Commons Reconocimiento 4.0 Internacional (CC BY 4.0).

ABSTRACT: Current developments of ecology have opened up new challenges for contemporary philosophical reflection. In this sense, it is not possible to interpret human nature starting from the idea of the human being as being isolated from its environment. The paradigm of human ecology presented here, therefore, aims to inherit the reflections developed in the philosophy of nature (and also in the philosophy of human nature), proposing the current ecological crisis as an eminently anthropological issue: man's place in the cosmos also indicates his essence. This task requires us to rethink the specificity of human beings in their relation to the world through the theme of $d$ welling, which invites us to reflect upon the activities of safeguarding and building the house. It thus becomes possible to consider the current ecological crisis as a predominantly anthropological crisis: to heal the wounds of the ecosystem, we must first heal the fracture within the human being itself.

KEYWORDS: Human Ecology; Human Nature; Environmental Ethics; Dwelling; Home. 


\section{UNA INTRODUCCIÓN HISTÓRICA NECESARIA}

La cuestión ecológica se encuentra en el centro de muchos debates contemporáneos. Desde que Ernst Heinrich Haeckel introdujo en el panorama científico el concepto de Ökologie (Haeckel, 1866), sin duda el tema ha suscitado el interés de académicos de diferentes disciplinas, desde la biología a la botánica, desde la zoología a la cibernética, desde la etología a la filosofía. El vínculo entre ecología y filosofía es muy sólido, no solo por los estudios de Haeckel -que era un famoso biólogo, zoólogo y filósofo- sino también porque la ecología con frecuencia ha demostrado invadir el ámbito de la reflexión cosmológica y antropológica.

El tema ecológico se ha convertido así en una cuestión filosófica. En los años setenta del siglo pasado, en los países anglosajones, se desarrolló la filosofía del medio ambiente $y$, con esta, la ética ambiental. Alrededor del final de los años cuarenta, sin embargo, con la publicación del famoso libro de Aldo Leopold A Sand Country Almanac (Leopold, 1949), ya se había visto la necesidad de una ética de la tierra (Land Ethic), capaz de actualizar las especulaciones anteriores de la filosofía de la naturaleza. Unos años más tarde, con la difusión de la obra de Rachel Carson, Silent Spring (Carson, 1962), la ética ambiental comenzó a ganar popularidad y la atención del público en general, y a utilizarse como un instrumento de denuncia de la conducta humana irresponsable hacia el ambiente. En los años setenta, finalmente, el tema ambiental se hizo de conocimiento público con la divulgación del informe del Club de Roma Limits to Growth (Meadows, Meadows, Randers y Beherns, 1972), donde se discutía el tema de la sustentabilidad, de importancia central en la filosofía ambiental. En esos mismos años (1972-1973) nació, gracias al filósofo noruego Arne Næss, el famoso movimiento de la Deep Ecology (Næss, 1973): este evento sin duda ha podido finalmente contextualizar la cuestión ecológica dentro de la especulación filosófica, liberándola en parte por la exasperada necesidad de datos científicos.

Hay que recordar también que el desarrollo de la ciencia ecológica ha fomentado en gran medida el nacimiento de otra disciplina muy actual: la bioética. Potter, considerado el padre de la misma ${ }^{1}$, de hecho destaca:

"Lo que ahora debemos enfrentar es que la ética humana no puede ser separada de un entendimiento realista de la ecología en el sentido más amplio de la palabra. Los valores éticos no pueden separarse de los hechos biológicos. Tenemos una gran necesidad de una ética de la Tierra, de una ética de la vida salvaje, de una ética de la población, de una ética del consumo, de una ética urbana, de una ética internacional, de una ética geriátrica, etc. Todos estos problemas requieren acciones que estén basadas sobre valores y hechos biológicos" (Potter, 2002, p. 122).

Es imprescindible, por lo tanto, tener en cuenta que gran parte de la especulación filosófica ambiental se configura como respuesta a la tendencia -propia del método científico- a reducir la naturaleza a un conjunto de símbolos y fórmulas matemáticas. Tal insatisfacción se dirige a la ciencia moderna, que parece constantemente buscar estructuras abstractas y universales en la naturaleza, sin comprender plenamente nuestra experiencia concreta de ser-en-el-mundo (Næss, 1989, p. 50). En este sentido, la ecología comparte la misma preocupación de la bioética: curar la herida creada por la ciencia moderna entre las dos culturas (Snow, 2009), creando un "puente" que pudiera poner en dialogo dos áreas cerradas en sí mismas. Así escribió Potter:

\footnotetext{
"Al sugerir una nueva disciplina llamada Bioética y al especificar que buscamos fundamentarla por fuera de las ciencias tradicionales, no estoy sugiriendo que abandonemos el tratamiento tradicional por una idea nueva, sino que atravesemos las fronteras disciplinarias más libremente y busquemos ideas que sean susceptibles de una verificación objetiva en términos de la futura supervivencia del hombre y el mejoramiento de la calidad de vida para futuras generaciones" (Potter, 2002, p. 126).

“Necesitamos", pues, "combinar la biología con el conocimiento humanista de distintas fuentes y forjar una ciencia de la supervivencia que sea capaz de implantar un sistema de prioridades" (Potter, 2002, p. 124).
}

Entre las razones para la afirmación conjunta de la bioética y de la ecología, además, hay que recordar la necesidad de replantear el papel -cada vez más invasivo- de la tecnología en nuestras vidas. El poder tecnológico ganado por el hombre en los últimos años, de hecho, parece haber traído consigo un cambio radical, no solo en la acción sino también en la misma concepción antropológica. Como ha destacado en varias ocasiones Hans Jonas, precursor de los debates ambientales contemporáneos, la tecnología ha cambiado al ser humano, transformándolo radicalmente; el hombre no es ya el sujeto creador, sino el objeto de la tecnología (Jonas, 1973, pp. 38-40) que él mismo un día había creado: 


\begin{abstract}
"La técnica, esa obra fríamente pragmática de la astucia humana, sitúa al hombre en un papel que solo la religión le había atribuido a veces: el administrador o guardián de la Creación. En tanto la técnica engrandece su poder hasta el punto en que se vuelve sensiblemente peligrosa para el conjunto de las cosas, extiende la responsabilidad del hombre al futuro de la vida en la tierra, que ahora está expuesta indefensa al abuso de ese poder" (Jonas, 1997, p. 36).
\end{abstract}

Ese mismo poder genera, por lo tanto, una nueva conciencia:

“Como especialista en cáncer, me impresionó naturalmente la afirmación de N. J. Berrill [...]. El observaba que: "en lo que concierne a la naturaleza, nosotros somos como un cáncer cuyas células extrañas se multiplican sin control, descaradamente, exigiendo el alimento que todo el cuerpo necesita [...]". En otras palabras, podemos hacernos la pregunta, ¿es el destino del hombre ser para la tierra viviente lo que el cáncer es para el hombre?" (Potter, 2002, pp. 123-124).

El movimiento de la ecología profunda ha contribuido en gran medida a la difusión de esta imagen negativa del hombre y de sus acciones ${ }^{2}$, definiendo "la crisis ecológica como el resultado del humanismo antropocéntrico que es fundamental para las principales ideologías modernas" (Zimmerman, 1994, pp. 1-2). Así, "el hombre podría ser descrito como un parasito altamente destructivo que amenaza destruir a su anfitrión -el mundo natural- y, finalmente, a sí mismo" (Bookchin, 2004, p. 23). Estos textos confirman las raíces comunes de la bioética y la ética ambiental, con referencia a la idea del hombre como el "cáncer del planeta" (Valera, 2014b), debido a su poder altamente destructivo:

"Nuestros problemas ambientales se originan en la arrogancia de imaginarnos como el sistema nervioso central o el cerebro de la naturaleza. No somos el cerebro, somos un cáncer de la naturaleza" (Foreman, 1990, abril, p. 48).

Estas son, pues, las principales razones de la necesidad de un enfoque filosófico sobre la cuestión ecológica, para entender la popularidad y difusión casi sincrónica de la bioética y de la ética ambiental. Simultáneamente al desarrollo de la ciencia ecológica, por lo tanto, la filosofía de la ecología ha intentado elaborar modelos interpretativos para explicar el significativo cambio de pensamiento inducido por el nacimiento de esta ciencia. Pero esta tarea se demostró muy difícil a causa del extraordinario crecimiento y propagación de una ciencia tan joven como la ecología: en los primeros años del siglo XX comenzaron a nacer distintas "ecologías", cada una con un objeto de investigación distinto; a la luz de este hecho, hoy podemos hablar de "ecologías" en plural (Valera, 2011, pp. 1026-1031). Entre estas, la ecología humana es particularmente interesante, ya que parece tener una inspiración filosófica más significativa, puesto que centra su reflexión en el papel principal que el ser humano interpreta en los cambios ambientales y climáticos. Si, de hecho, la ecología es la ciencia que estudia las relaciones existentes entre los organismos vivientes y el ambiente, la ecología humana -al menos en el proyecto embrionario de Gerald Marten, uno de los iniciadores de la disciplina- trata de las relaciones entre los seres humanos y sus ambientes (Marten, 2003, p. 1). La ecología humana analiza, así, las consecuencias de las actividades humanas como una cadena de efectos entre el ecosistema y el sistema social humano. Por lo tanto, la centralidad de la ecología humana entre las distintas ecologías deriva del hecho de que todas las especies se adaptan al ambiente, excepto la especie humana, que adapta el medio ambiente a sus exigencias y deseos.

\section{ECOLOGÍA HUMANA: BUSCANDO UNA DEFINICIÓN}

Antes de presentar nuestra propuesta relativa a un nuevo paradigma de ecología humana, hay que examinar los supuestos de los principales pensadores que contribuyeron al debate sobre esta "disciplina", destacando los aspectos positivos y los puntos débiles.

Terry Rambo (1983, p. 1) escribe: "La ecología es la ciencia de las relaciones entre los organismos vivientes y el medio ambiente", y, más específicamente, "La ecología humana es el estudio de las interacciones humanas con el ambiente". Debido a las formas singulares en las que las personas modifican el medio ambiente, la ecología humana es, entonces, central entre las distintas ecologías. La ecología humana ha alcanzado una considerable relevancia práctica en nuestro tiempo; por ejemplo, ha contribuido en gran medida a la definición de los criterios para los proyectos urbanos y la planificación económica (Lima, 2000). La ecología humana ha hecho uso de los cambios históricos, geográficos, médicos, económicos y sociales que han ayudado a lograr un marco completo y articulado para las diversas necesidades humanas, que antes considerábamos solo a nivel parcial; ha partido de una variedad de disciplinas como la geografía económica, la higiene y la epidemiología. 
Gerald Marten, uno de los investigadores más importantes en ecología humana, escribe:

"El medio ambiente, en la ecología humana, se percibe como un ecosistema. Un ecosistema es todo lo que existe en un área determinada -el aire, el suelo, el agua, los organismos vivos y las estructuras físicas, incluyendo todo lo construido por el ser humano-. [...] Aunque los seres humanos son parte del ecosistema, es útil pensar en la interacción de los seres humanos y el ecosistema como la interacción del sistema social humano y el resto del ecosistema. [...] El sistema social es un concepto central en la ecología humana, porque las actividades humanas que ejercen algún impacto sobre los ecosistemas están fuertemente influenciadas por la sociedad en que viven las personas. Los valores y conocimientos [...] determinan la manera en que procesamos e interpretamos la información y cómo la traducirnos en acción. La tecnología define nuestro repertorio de acciones posibles" (Marten, 2003, pp. 1-2).

Por un lado, Marten tiende a alejarse de una visión reduccionista de la relación del ser humano con el medio. Por otro lado, sin embargo, él parece conservar una perspectiva sociobiologista, que interpreta la humanidad como fuente de valores y experiencias sociales $y$, en este sentido, que no puede exceder la dimensión cuantitativa universal, hacia la afirmación de la singularidad cualitativa de cada persona humana. La necesidad de afirmar la singularidad cualitativa humana -dada por la libertad- queda, por lo tanto, abierta y sin solución (Bruckmeier, 2013, pp. 229-234).

Así pues, también la ecología humana, si es caracterizada de manera simplista como "el esfuerzo de comprender las relaciones entre las personas y su medio ambiente" (Bates y Tucker, 2010, p. 4), puede caer en dos tentaciones distintas: la tentación reduccionista de interpretar al ser humano como agente únicamente bío-social; y en segundo lugar, la tentación sociologista, que no explica cómo el hombre debe actuar en el ambiente -y por lo tanto cómo el hombre debería ser-sino que más bien describe la forma en que de hecho lo hace. Tales tentaciones pueden verse ya en las primeras investigaciones en este ámbito:

"La ecología humana es, fundamentalmente, el esfuerzo por investigar: 1. Los procesos mediante los cuales se mantiene el equilibrio biótico y social [...];

2. Los procesos mediante los cuales, si el equilibrio biótico y social se interrumpe, puede tener lugar una transición de un orden relativamente estable a otro" (Park, 1936, p. 15).
La posibilidad para la ecología humana de evitar el reduccionismo darwiniano y sociobiologista solo viene garantizada por un replanteamiento de la complejidad humana a través de instrumentos antropológicos y filosóficos (Beltrão, 1985). Una ecología humana sin reduccionismo requerirá una reflexión más profunda sobre la relación entre el ser humano y su ambiente y, por otra parte, sobre la relación que el ser humano tiene consigo mismo. Sin un cambio tal de actitud tendremos que conformarnos con una nueva disciplina que no logra captar la especificidad humana, por más que haga de la interdisciplinariedad su distintivo:

"La ecología humana puede ser vista como la génesis de una nueva disciplina científica, una reflexión sobre la ciencia y sus valores, o como una manera de acercarse a las distintas sociedades. Más específicamente, la ecología humana, a partir de diferentes enfoques de distintas disciplinas, ha desarrollado su propia metodología de estudio, considerando la dinámica de las interacciones bioculturales en los ecosistemas. Esta actitud científica de integración entre las diferentes disciplinas le permite explorar los campos del conocimiento a través del estudio del equilibrio dinámico de los ecosistemas en los que está implicado el hombre" (véase Certificat International d'Écologie Humaine (CIEH)).

Sin embargo, la ecología humana nació precisamente en oposición a las tendencias anti-antropocéntricas y utilitaristas que caracterizan la ecología profunda y superficial (Valera, 2014b). De hecho, la ecología humana nació como un conocimiento cuyo objetivo es el de devolver la especie humana al centro del universo moral, con la esperanza de reconstruir la relación correcta de los seres humanos consigo mismos y con otras especies. Los seres humanos pueden ser considerados no solo como un cáncer del planeta, sino también como la posible cura: en este sentido, la ecología humana cree en los seres humanos y en sus capacidades de construir y de desarrollar nuevas soluciones.

Las soluciones propuestas por la ecología humana, de hecho, ponen énfasis en la libertad del individuo como base para crear un mundo más sostenible, y tratan de evitar la limitación de la libertad de los individuos en nombre de la reducción de su impacto ambiental:

"En nuestro interés de preservar la libertad, ignoramos la larga y dolorosa lucha por la que esta se logró [...]. En nuestro deseo de dar a cada individuo las mismas oportunidades, nos hemos olvidado de 
aprovechar las diferencias entre los individuos que deben enriquecer nuestra sociedad. [...] Nos aseguramos los mejores entrenadores para los caballos y perros prometedores, pero permitimos que nuestros estudiantes más capaces se paren a causa de nuestro sistema escolar. [...] Deploramos el aumento de la delincuencia juvenil, pero hacemos poco para combatir la indolencia y la falta de motivación" (Sears, 1954, p. 960).

Si la especie humana es, de hecho, la especie que, más que cualquier otra, puede cambiar el entorno que la rodea, hay que comenzar precisamente a partir de ella para encontrar soluciones prácticas y sostenibles (Valera y Marcos, 2014), de modo que su relación con las otras especies puede llegar a ser virtuosa y no perjudicial. Por lo tanto, los "objetos" de la ecología humana son principalmente los individuos y las relaciones entre los seres humanos y, después, la relación de la humanidad con el medio.

Solamente a la luz de esto, el conocimiento propuesto por la ecología humana podrá ser auténticamente interdisciplinario y tendrá como objetivo integrar los conocimientos de las disciplinas científicas con los de las humanidades, para tener una mejor comprensión general de las dinámicas que afectan a los seres humanos y que los motivan a actuar de ciertas maneras. Por esta razón, la "ecología humana se ocupa no solo del presente, sino también del pasado. [...] Y, puesto que el presente y el futuro de cualquier comunidad son expresiones de su pasado, podemos esperar que el estudio de la arqueología y de la historia tengan un efecto práctico en la cuestión fundamental del futuro del hombre" (Sears, 1954, p. 960).

La combinación de dos tipos diferentes de conocimiento -ciencia ecológica y humanidades- permitirá el descubrimiento de la dimensión humana, que es esencial para la preservación sostenible de nuestro planeta:

\footnotetext{
“¿Quién mejor puede analizar y explicar tales condiciones que el ecologista, entrenado como debe ser para leer el paisaje? Pero debe estar equipado también para analizar la comunidad humana y para comprender las fuerzas que actúan en ella" (Sears, 1954, p. 963).
}

En este sentido, la ecología humana tiene que ver con las relaciones humanas, en sus tres direcciones principales: 1) la relación que los seres humanos construyen con su entorno (el impacto de la agricultura en el ecosistema, las consecuencias de las distintas opciones tecnológicas, las intervenciones a emprender, etc.); 2) la relación que el ser humano individual entreteje con los otros seres humanos (la posibilidad de compartir las necesidades, la búsqueda de soluciones y de políticas adecuadas, etc.); y 3) la relación que cada individuo humano construye en diálogo consigo mismo (las virtudes que puede desarrollar, los valores esenciales, la prioridad asignada a los diferentes bienes, etc.). Hay que señalar que negar esta última dimensión significa negar la ecología humana misma, y, en última instancia, significa dar apoyo a la ecología profunda o a la ecología superficial, según las cuales los seres humanos no pueden ser la solución a la crisis ecológica actual.

\section{EL PUNTO DECISIVO DE LA ECOLOGÍA HUMANA: LA ECOLOGÍA ES UNA ANTROPOLOGÍA}

En el nacimiento y desarrollo de la ecología humana destaca una necesidad, muy fácilmente olvidada por el pensamiento ecológico: existe un vínculo indisoluble entre la ecología y la antropología, es decir, entre una determinada concepción del mundo y una visión del hombre. Por lo tanto, la clave ecológica es el ser humano, y el comportamiento ecológico solo puede ser el comportamiento humano.

Por el contrario, no es acertada la idea de que la reflexión sobre el ser humano puede (o debe) ser separada de la reflexión sobre el medio ambiente (y viceversa) y, en consecuencia, de que la ecología como ciencia tiene por objeto exclusivo el "entorno" del hombre, y no el hombre mismo. Hoy en día esa parece ser la posición predominante en la ecología: se tiende a hipostasiar el medio ambiente, dándole vida, "conciencia" y dignidad autónoma, independiente del ser humano, como si una seria reflexión sobre el ambiente debiera necesariamente poner entre paréntesis al hombre. La epoché de la ecología, herramienta que se utiliza para "limpiar" el medio ambiente de las escorias "artificiales" humanas, es sin embargo artificiosa. Artificiosa, ya que es reduccionista (se reduce al hombre a un cuerpo que interactúa con otros cuerpos); artificiosa, ya que es ahistórica (¿existe un entorno sin el hombre, sin su intervención?); y artificiosa, ya que es antiecológica (¿la libertad humana no es una dimensión ecológica?).

Hoy también la ética ambiental, habiendo perdido a su actor principal, el ser humano -de hecho, el único actor capaz de juicio ético-, se consuela con muchos "actores" secundarios, como los animales no humanos, las plantas, Gaia, etc. Pero, aunque un ambiente sin hombre podría quizás ser posible, una ética ambiental, no. Y lo mismo pasa con la ecología: 
el único ser capaz de llevar a cabo un discurso sobre el medio ambiente y actuar con intencionalidad es, de hecho, el ser humano.

La ecología gana cuando considera al ser humano como fundamental y único posible promotor de un discurso sobre la casa (oikos-logos), pasa así de ser una ciencia meramente descriptiva del medio ambiente a convertirse en una reflexión -con implicaciones normativas- sobre el ambiente, sobre los seres humanos y sobre las relaciones de estos con su entorno y consigo mismos.

El paso decisivo para ganar esa perspectiva es abrazar simultáneamente la idea de la "ecología" y la idea de lo "ecológico". Las dos dimensiones parecen, en principio, incomunicables: si la primera trata de las relaciones dentro del ecosistema, la segunda refiere a un predicado atribuible a los comportamientos humanos. La discusión aquí requiere algunas aclaraciones, en particular en lo que se refiere al término ecológico: ¿cuál es su significado? $\mathrm{Y}$, de nuevo, ¿a quién se refiere? A menudo tendemos a sostener que un tren es ecológico, que un determinado modo de producción puede ser ecológico, que un gesto tiene que ser ecológico, etc. Tres referentes diferentes, que al parecer no presentan ningún elemento de similitud: un producto técnico, una actividad y un comportamiento; una mejor observación nos muestra, de hecho, que el elemento común entre las tres es el mismo ser humano: es el hombre quien construye el producto técnico, es el hombre quien actúa, y es el hombre quien se comporta.

El adjetivo ecológico connota siempre -incluso en el caso del tren, que está construido y puesto en acción por un ser humano- una acción humana: por esta razón el estilo de la ecología humana es muy similar al estilo de la ética. Por lo tanto, el árbol no puede ser ecológico, ya que no puede comportarse de una manera respetuosa para con el medio ambiente: el árbol es, no hace. Y lo mismo cabe decir respecto de los animales no humanos. En este sentido, creemos que Heidegger describe adecuadamente la diferencia entre los seres humanos y los otros seres cuando afirma que "la piedra es sin mundo, el animal es pobre en mundo, el hombre es configurador de mundo" (Heidegger, 2000, p. 152). También es cierto que existe una gradación en el "ser ecológicos", así como en el bien: como una acción es más o menos buena, de la misma manera puede ser más o menos ecológica.

Por estas razones, la propuesta que planteamos, para superar las dificultades previas de definición, es la de referirse a la ecología humana como a un estudio crítico, que tiene sus raíces en el terreno de la ética, de la antropología filosófica y de la cosmología. No sabemos si sería apropiado conferir a la ecología humana el papel de ciencia: por ahora preferimos definirla cautelosamente como reflexión crítica sobre la relación del hombre con el medio ambiente y con el hombre mismo. En este sentido, la ecología humana lleva a consideraciones éticas, antropológicas y cosmológicas, y tiene como objetivo definir cómo esta relación debe ser, no solo como es.

\section{UNA ANTROPOLOGÍA RELACIONAL PARA LA ECO- LOGÍA HUMANA: HABITAR LA CASA}

La dicotomía contemporánea entre antropocentrismo y biocentrismo, de hecho, ha permitido la emergencia de un problema desde hace siglos olvidado por la antropología filosófica, la cual se había ocupado, sobre todo, de resolver la controvertida cuestión de la relación entre alma y cuerpo: el problema del puesto del hombre en el cosmos. Escribe Max Scheler en el prólogo a la primera edición del famoso libro:

\footnotetext{
"Las cuestiones: '¿Qué es el hombre? y ¿cuál es su puesto en el ser?', me han ocupado de un modo más directo y esencial que todas las demás cuestiones de la filosofía, desde el primer despertar de mi conciencia filosófica" (Scheler, 1964, p. 19).
}

Las respuestas dadas por el paradigma biocéntrico y por el antropocéntrico a estas cuestiones no parecen exhaustivas: el primero, sin embargo, ha puesto de nuevo al hombre en el ambiente, haciéndolo así permeable y vulnerable a las dinámicas sistémicas del ecosistema (Marcos, 2012a, p. 109); el segundo, por el contrario, ha distanciado al hombre de la naturaleza y lo ha puesto en una dimensión totalmente diferente, por lo que el hombre ha olvidado su naturaleza, incluso su naturaleza animal. Más allá de estas dos respuestas, que no compartimos en cuanto demasiado extremas, parece interesante volver a la pregunta inicial: ¿dónde estamos? Y aún más: ¿dónde debemos estar? Si la primera pregunta revela una cuestión que tiene sus raíces en la ecología, la segunda, al parecer, no. Hay, por lo tanto, que poner temporalmente entre paréntesis la cuestión esencial acerca de qué es el hombre, para dar paso a la pregunta aparentemente más neutral sobre el espacio que el ser humano concreto puede y debe ocupar. La concepción del espacio aquí evocada es mucho más amplia que la mera noción física, ya que incluye también la dimensión temporal: la antropología filosófica contemporánea, de matriz esencialmente histórica, tiene el gran mérito 
de haber colocado de nuevo el ser humano en un espacio físico y temporal determinado (Valera, 2014a), el mérito de referirse siempre al hombre "hic et nunc".

Con una mirada superficial se podría quizá concluir precipitadamente que las preguntas sobre el lugar propio del hombre (Wo ist der Mensch?) son capaces de ocultar la pregunta sobre la esencia del mismo (Was ist der Mensch?), pero no es así. El testimonio de la coexistencia de las dos preguntas se manifiesta en la obra de Scheler:

\footnotetext{
"Los problemas fundamentales de la filosofía pueden reducirse a la pregunta ¿qué es el hombre? ¿Cuál es el lugar y posición dentro del ser, del mundo, y de Dios?" (Scheler, 1960, p. 37).
}

Hay que tener en cuenta también que la pregunta sobre el lugar del hombre en el mundo siempre ha tenido que ver con su esencia. Ya al comienzo de la historia humana, a la pregunta de Dios acerca del lugar que el hombre ocupa en el mundo (“¿dónde estás?”), Adán responde con un estado de ánimo ("estoy en el miedo"), como para subrayar esta conexión original entre el espacio y la esencia:

“Dios el Señor llamó al hombre y le preguntó: ‘¿Dónde estás?'. El hombre contestó: 'Escuché que andabas por el jardín y tuve miedo, porque estoy desnudo; por eso me escondí'". (Génesis, 3, 9-10).

La reflexión ecológica contemporánea es, pues, hija de la modernidad: la separación de las dos preguntas acerca del hombre ha llevado a la elaboración de paradigmas incomunicables $y$, por lo tanto, mutuamente incomprensibles. La pregunta "¿quién es el hombre?" -que a causa del pensamiento darwinista se ha convertido en la pregunta "¿qué es el hombre?"- no tiene nada que ver con la pregunta acerca del lugar del hombre, porque no se considera jamás al hombre como sujeto. Si, en última instancia, el mérito del pensamiento ecológico contemporáneo es el de abordar la cuestión del puesto del hombre en el cosmos, su mayor defecto es el de ocultar la cuestión sobre la esencia, separando así las dos preguntas.

Las dos preguntas, sin embargo, no están realmente separadas, ya que al final apuntan a dos aspectos diferentes -pero no mutuamente excluyentes- de la misma realidad del ser-hombre: el hombre que vive más allá del tiempo y del espacio y el hombre que vive en ellos. De otra manera: se podría afirmar que la historicidad del hombre es una parte constitutiva de su esencia. Sin el “¿quién?" no hay “¿dónde?”, y viceversa. Fuera de la historia no existe el hombre.
De una manera más radical que la nuestra, Ortega y Gasset sugiere que el hombre no tiene naturaleza, sino historia (Ortega y Gasset, 1983, p. 41). Sin embargo, hay que tener cuidado con el uso de los términos y con el significado de las palabras: el hombre, al igual que el animal no humano, tiene historia; pero el hombre, a diferencia del animal no humano, es historia. Ya en el hecho de tener historia hay diferencias entre el ser humano y el animal no humano. El hombre tiene historia como "auto-reflexión, autoconocimiento y auto-comprensión, el animal no humano, no. El hombre se entiende como ser histórico, el animal no humano es entendido como ser en la historia. Además, el ser humano es su propia historia, ya que la historia depende de sus elecciones y de su libertad. Se podría decir que el ser humano es la historia de su propia iniciativa libre en el medio ambiente y en sí mismo, es decir, es la historia de su progresiva liberación de la esclavitud del instinto y de la reacción al ambiente. En pocas palabras, podríamos concluir que el hombre tiene la historia que es, mientras el animal es la historia que tiene. La historia de la libertad es, pues, radicalmente diferente de la historia del ambiente, ya que coincide con la historia de la conquista del mundo, es decir, con su habitación.

\section{HABITAR LA CASA: LA ECOLOGÍA HUMANA COMO ETHOS}

La construcción de la propia humanidad, que procede de la objetivación del entorno de la cual solo un ser racional como el ser humano es capaz, es propiamente el habitar:

"Ahora, el mejor término que hereda la reflexión [...] acerca del 'lugar' -cuando eso se refiere al hombre- es 'habitar'. El hombre no vive en un mero ambiente, no ocupa un puro espacio y no se instala simplemente en un sitio, sino que siempre habita: el hombre existe como hombre en cuanto habita un lugar" (Petrosino, 2007, p. 10).

El hombre vive en el mundo, ya que está en el mundo y al mismo tiempo no es del mundo: el hombre habita. De esta manera, el ser humano es parte de la naturaleza, pero no está determinado por la naturaleza:

"Los mortales son; esto quiere decir: habitando aguantan espacios sobre el fundamento de su residencia cabe cosas y lugares. Y solo porque los mortales, conforme a su esencia, aguantan espacios, pueden atravesar espacios. Sin embargo, al andar no abandonamos aquel estar [...]. Yo nunca estoy sola- 
mente aquí como este cuerpo encapsulado, sino que estoy ahí, es decir, aguantando ya el espacio, y sólo así puedo atravesarlo" (Heidegger, 1994, pp. 137-138).

Por lo tanto, el habitar es un gesto exclusivo del hombre, y "pertenece a cada hombre, en la medida en que, simplemente no se somete a la vida, vuelve con insistencia sobre ella, se flexiona sobre ella (reflexiona) con el fin de re-tomarla, entenderla, dominarla, y, en última instancia, nombrarla" (Petrosino, 2007, p. 10). El habitar es el signo del dominio del hombre sobre la naturaleza, que transforma las cosas naturales en objetos, en entidades nombrables y entonces comprensibles. Heidegger escribe:

"El espaciar aporta lo libre, lo abierto para un asentamiento y un habitar del hombre. Pensando en su propiedad, espaciar es libre donación de lugares, donde los destinos del hombre habitante tornan forma en la dicha de poseer una tierra natal o de la desgracia de carecer de una tierra natal, o incluso en la indiferencia respecto a ambas [...]. Por un lado, el emplazar admite algo. Deja que se despliegue lo abierto que, entre otras cosas, permite la aparición de las cosas presentes a las cuales se ve remitido el habitar humano. Por otro lado, el emplazar proporciona a las cosas la posibilidad de pertenecerse mutuamente, estando cada una en su respectivo sitio y desde donde se abre a las otras cosas" (Heidegger, 2009, pp. 21-23).

En este sentido, entonces, el hombre es también el constructor del mundo:

"En la construcción, cuando él construye como hombre, el hombre nunca erige constructos simples, pero [...] él también define un lugar y establece un mundo, es decir circunscribe un espacio" (Petrosino, 2003, p. 47).

La capacidad de objetivar, dominar y nombrar las cosas indica la capacidad del hombre de distanciarse de ellas, ganando una posición que no está necesariamente enredada en ellas. El hombre, por lo tanto, habita el mundo a medida que descubre en la naturaleza un significado, que siempre la trasciende. Así dice Scheler:

"Cuando el hombre se ha colocado fuera de la naturaleza y ha hecho de ella su 'objeto' -y ello pertenece a la esencia del hombre y es el acto mismo de la humanificación- se vuelve en torno suyo, estremeciéndose, por decirlo así, y pregunta: ‘¿Dónde estoy yo mismo? ¿Cuál es mi puesto?' El hombre ya no puede decir con propiedad: 'Soy una parte del mundo; estoy cercado por el mundo'; pues el ser actual de su espíritu y de su persona es superior incluso a las formas del ser propias de este 'mundo' en el espacio y en el tiempo. En esta vuelta en torno suyo, el hombre hunde su vista en la nada, por decirlo así. Descubre en esta mirada la posibilidad de la 'nada absoluta'; y esto le impulsa a seguir preguntando: '¿Por qué hay un mundo? ¿Por qué y cómo existo 'yo'?" (Scheler, 1964, pp. 108-109).

Queda difuminado así el forzado y artificioso conflicto entre el hombre y el mundo, entre el hombre y la naturaleza, entre el hombre y el espacio:

"Cuando se habla de hombre y espacio, oímos esto como si el hombre estuviera en un lado y el espacio en otro. Pero el espacio no es un enfrente del hombre, no es ni un objeto exterior ni una vivencia interior. No hay los hombres y además espacio; porque cuando digo "un hombre" y pienso con esta palabra en aquel que es modo humano, es decir, que habita, entonces con la palabra "un hombre" estoy nombrando ya la residencia en la Cuaternidad, cabe las cosas" (Heidegger, 1994, p. 137).

Esto lleva también al fracaso de las posiciones biocéntricas y antropocéntricas, que aparecen ahora como inadecuadas y reduccionistas, ya que no son capaces de entender al humano como ser del mundo y no solo como ser en el mundo.

Si, entonces, habitar es la condición para que el hombre esté en el mundo -Heidegger diría: un hombre que no habita es una contradicción viviente, ya que "habitar no es una de las prácticas del hombre, sino el carácter fundamental de la misma naturaleza humana" (Pesare, 2006, p. 7)-, ¿qué significa, en última instancia, habitar?:

"El rasgo fundamental del habitar es este cuidar (mirar por) [...]. Los mortales habitan en la medida en que salvan la tierra [...]. La salvación no sólo arranca algo de un peligro; salvar significa propiamente: franquearle a algo la entrada a su propia esencia. Salvar la tierra es más que explotarla o incluso estragarla. Salvar la tierra no es adueñarse de la tierra, no es hacerla nuestro súbdito, de donde sólo un paso lleva a la explotación sin límites" (Heidegger, 1994, pp. 131-132).

$\mathrm{Si}$ "el habitar es más bien siempre un residir cabe las cosas" (Heidegger, 1994, p. 133), esta actitud transforma el mundo en una morada (ethos), a través de la acción del cuidar: emerge así la dimensión ética del demorar. La relación -que parecería ser puramente nominal- dada por la etimología de ethos (al mismo tiempo: 'comportamiento', 'conducta' y 'morada'), se convierte, por lo tanto, en relación "sustancial" a partir del cuidado: el cuidar como dimensión funda- 
mental del habitar el mundo y el cuidado como una dimensión esencial de la apertura a la alteridad.

En este sentido, la ética en su sentido propio y original corresponde a la "forma de estar en el mundo" $y$, por tanto, alude a un enraizamiento, a una pertenencia a algo que de alguna manera nos precede siempre. Desde este punto de vista, la ética, antes de tener que ver con el deber, identifica las formas de vida que también son visiones del mundo, convicciones, creencias (Natoli, 1999).

Si la característica fundamental del habitar es el cuidar y la forma de estar en el mundo es la ética, entonces cuidar es el primer modo de enfoque ético sobre mundo, su dimensión más íntima y distintiva. El hombre puede realizar la ecología humana ya que es capaz de cuidar el mundo, ya que puede "salvarlo", es decir, liberarlo de la brutalidad del instinto.

El mundo, sugiere Heidegger, es dado al hombre en cuanto él es el único ser capaz de "liberar su esencia", que solo él puede entender: por primera vez reaparece en la contemporaneidad la posibilidad de que el ser humano, de un simple destructor (la "explotación ilimitada" es siempre posible), pueda llegar a ser el liberador, el salvador. La pregunta que puede surgir de forma espontánea, a la vista de estas reflexiones, es: ¿por qué, entonces, vemos una crisis, desde hace más de un siglo, en la relación entre los seres humanos y la naturaleza?, ¿por qué nuestra vida en el mundo (la casa, oikos) ya no es, en última instancia, determinada por el cuidado, sino, más bien, por el abandono? Así contesta Heidegger:

"La auténtica penuria del habitar no consiste en primer lugar en la falta de viviendas [...]. La auténtica penuria del habitar descansa en el hecho de que los mortales primero tienen que volver a buscar la esencia del habitar, de que tienen que aprender primero a habitar. ¿Qué pasaría si la falta de suelo natal del hombre consistiera en que el hombre no considera aún la propia penuria del morar como la penuria? Sin embargo, así que el hombre considera la falta de suelo natal, ya no hay, entonces, más miseria. Aquélla es, pensándolo bien y teniéndolo bien en cuenta, la única exhortación que llama a los mortales al habitar" (Heidegger, 1994, p. 142).

La crisis ecológica es, en primer lugar, una crisis del habitar y, por eso, una crisis del modo de relación entre yo/tú. La crisis del habitar es también una crisis del pensamiento sobre la naturaleza humana (sobre su esencial enraizamiento) y sobre las relaciones humanas. La liberación ecológica, la liberación de la crisis ecológica -traducción y actualización de las palabras de Heidegger- tiene que ser, entonces, una liberación únicamente humana, que surge de la conciencia de la naturaleza humana y del mundo.

\section{OIKOS-LOGOS: LA ECOLOGÍA HUMANA COMO CONSTRUIR Y CUSTODIAR LA CASA}

Por otro lado, la crisis ecológica es, al mismo tiempo, el reflejo de la crisis de la casa en cuanto morada. El concepto de 'casa' -más que un concepto es propiamente una experiencia- es extremadamente denso y difícil de entender: habla de lo incalculable, de lo inconmensurable. Habla de una intimidad que se configura como inaccesible a cualquier instrumento de precisión, ya que, en última instancia, se basa en principios que no son reducibles a los meros hechos y que tampoco son observables:

"En pocas palabras, intimidad significa la oportunidad de ser recibido [...] sin miedo, sin vergüenza, sin censuras o autocensuras, de estar 'desnudo'. [...] La manifestación plena de la humanidad demanda, o tal vez mejor espera, un lugar íntimo donde se puede estar 'desnudo'" (Garlaschelli y Petrosino, 2012, pp. 26-27).

La intimidad, en cuanto lugar de la desnudez, se caracteriza como un delicado espacio límite entre la observabilidad del cuerpo vivo y la invisibilidad de la persona y, al mismo tiempo, como la condición de posibilidad de la apertura al otro. Si la casa -hogar de la intimidad- es el lugar de acogida, siempre se espera al otro, y nunca hay solo un "yo", sino un "yo-con-otros". Es interesante recordar aquí el concepto de lugar de Marc Augé, que evoca el tema de la casa:

"El lugar antropológico se define en primer lugar como el lugar de chez soi, en casa, el lugar de la identidad común" (Augé, 2007, p. 42).

Por lo tanto, es esta apertura la que define correctamente la casa como un lugar humano, como una dimensión personal y nunca animal. De esta manera, la casa no puede ser una guarida:

“En la 'casa' hay umbrales, siendo justo lo que se recoge y se cierra alrededor de una abertura irreductible. [...] Ahora, la guarida es el fruto de esa ilusión y perversión que quiere poner en seguridad absoluta todos los umbrales de un hogar" (Garlaschelli y Petrosino, 2012, p. 70).

Ese sentido de 'casa' es mucho más amplio y al mismo tiempo no se puede reducir a una arquitectura o topología: la casa no se identifica con las cuatro paredes, sino que más bien describe los espacios del habi- 
tar, es decir, de la manera esencial de ser "hombres en el mundo". En este sentido, la tierra puede convertirse en un hogar para los seres humanos, es decir en un lugar donde las relaciones se desarrollan y se convierten en una oportunidad de enriquecimiento para el hombre. Por otro lado, existe también la posibilidad de que el hombre, con su propio habitar, cree el desierto, o deje sin sentido un lugar (el campo de exterminio es un buen ejemplo de esta actitud). Incluso en este último caso, sin embargo, emerge con fuerza la posibilidad del impacto singular que el hombre tiene sobre el mundo: todas las posibles "desrealizaciones" o "desertificaciones" siguen siendo la expresión de una cierta manera de habitar y, en consecuencia, se acompañan inevitablemente con una producción de sentido y con la configuración de un lugar. Jean-Luc Nancy escribe:

\footnotetext{
“El crecimiento mismo del desierto podría desvelamos un espacio desconocido, una aridez desconocida, excesiva, de las fuentes del sentido" (Nancy, 1993, p. 44).
}

La desertificación de la realidad -"el desierto que crece"- es, pues, una consecuencia de una manera de habitar, de un vivir que ha olvidado el significado del habitar, en cuanto el habitar implica tanto un custodiar como un construir. Estas dos dimensiones (custodiar y construir) son los elementos esenciales del habitar.

Destacar el papel de constructor del hombre significa, en primer lugar, valorar las propiedades creativas e inventivas que lo distinguen, su deseo de cambiar su entorno, para adaptarlo a sí mismo en lugar de adaptarse, para encontrar nuevas soluciones. El hombre es constructor en cuanto que es señor (dominus) y puede ser dueño de las cosas, puede hacer uso de su ingenio, usar las cosas para sus propios fines $y$, por lo tanto, puede convertirlas en medios. El ingenio humano establece la superioridad del hombre sobre las demás criaturas vivientes.

Por otro lado, entonces, el hombre que habita en la naturaleza debe ser muy consciente de su profunda dependencia del mundo, aunque el mundo contemporáneo diga lo contrario: dependencia de un mundo sin el cual él no podría existir y, al mismo tiempo, dependencia de las relaciones con otras personas, a partir de su corporalidad, con demasiada frecuencia límite infranqueable. Es dentro de esta dinámica de dependencia (Marcos, 2012b; Valera, 2015b, p. 1457) donde surge la necesidad de custodia, como una forma responsable de vivir la relación con todo lo que es diferente del ser humano.
El custodiar y el construir no parecen, entonces, dos actitudes opuestas, sino dos aspectos de la misma actividad del habitar, dirigida al desarrollo y a la mejora del mundo. La unidad de las dos dimensiones también se propone como un antídoto eficaz para liberar de las tendencias radicales del conservadurismo y del progresismo, volviendo a poner en el centro del esfuerzo humano el objetivo de un desarrollo verdaderamente sostenible. La dimensión humana del habitar se refiere en última instancia a la alteridad como criterio:

"El hombre, habitando, ¿qué o quién es llamado a custodiar? En primera instancia, y, en un cierto sentido correctamente, se puede contestar que el custodiar afecta a la existencia y a la vida en general: el hombre, a diferencia de cualquier otro ser viviente, no sólo hace uso de los otros seres vivientes con el fin de vivir, sino que también está llamado a hacerse cargo de todos los demás seres vivientes y por lo tanto de la vida misma. [...] Sólo el hombre puede hacerse cargo de la alteridad. Y donde existe la cura, siempre hay alteridad. En este sentido, el concepto de cuidar no se entiende fuera de un horizonte de alteridad: el cuidar es siempre relativo a la alteridad del otro. [...] De hecho, todo lo que existe está relacionado con el otro (el ser es ser-con); todo lo que vive, simplemente para mantener su vida entra en una relación con los demás, es decir se abre al otro (el ser es inter-és); pero sólo el hombre hace la experiencia del otro como otro, es decir, como un ser irreductible a sí mismo (el otro es des-interés)" (Garlaschelli y Petrosino, 2012, p. 39).

Existe, por lo tanto, una primera dimensión de relacionalidad inscrita en el ser mismo: la vida es siempre constitutivamente relacionada y un ser vivo para mantenerse vivo debe aceptar a los demás; sin embargo, el hombre acepta (o rechaza) la alteridad de una manera totalmente diferente de los demás seres vivientes: crea las condiciones de posibilidad para que el otro pueda revelarse como realmente "otro", es decir, como un "tú". La personalidad es diferencia y la alteridad es el signo de esta. La casa se convierte así en un producto finito de relaciones infinitas: dentro de espacios y recursos limitados, nacen una infinidad de relaciones que requieren nuevos espacios que no son medibles.

En este sentido, la ecología humana puede considerarse como la promotora de las relaciones (de la casa): la condición necesaria de la vida es la apertura, es su custodia, y es el reconocimiento de una alteridad que nos pide construir. Solo a partir de este reconocimiento se puede concebir cualquier relación (la relación 
con el árbol o con el animal no humano) y cualificarla como verdaderamente ecológica, sin el temor de ser injustos o políticamente incorrectos.

En última instancia, la importancia de la ecología humana reside en la posibilidad de rescatar al mismo tiempo la reflexión ecológica y la filosófica, retomando de nuevo la cuestión de la esencia del ser humano, sin olvidar el hecho de que el hombre es un ser constitutivamente relacional. La ecología humana hereda, así, la reflexión filosófica milenaria sobre la naturaleza humana, en constante diálogo con los nuevos descubrimientos en el ámbito de la ecología, desafiando al hombre contemporáneo a reconsiderar su esencia y a buscar nuevos paradigmas que expliquen de una manera más adecuada su puesto en el cosmos.

\section{AGRADECIMIENTOS}

El presente artículo forma parte del proyecto "CONICYT - Iniciación en Investigación Fondecyt 2016 - n. 11160113".

\section{NOTAS}

1. Aunque se sabe que la bioética, como disciplina, nació por la intuición de Van Rensselaer Potter, la primera aparición del término se encuentra en un texto del pastor protestante Fritz Jahr. En este texto se ve la profunda conexión entre la ecología y la bioética, ya que pone fuerte énfasis en la necesidad de ampliar el límite de nuestras consideraciones morales a todos los

\section{BIBLIOGRAFÍA}

Augé, M. (2007). Tra i confini. Città, luoghi, integrazioni. Milano: Bruno Mondadori.

Bates, D. G y Tucker, J. (2010). Human Ecology: Contemporary Research and Practice. New York: Springer. https://doi. org/10.1007/978-1-4419-5701-6

Beltrão, P. C. (1985). Ecologia umana e valori etico religiosi. Roma: Editrice Pontificia Università Gregoriana.

Bookchin, M. (2004). Post-scarcity anarchism. Oakland: AK Press.

Bruckmeier, K. (2013). Natural resource use and global change. New interdisciplinary perspectives in social ecology. Basingstoke: Palgrave Macmillan. https:// doi.org/10.1057/9781137303158

Carson, R. (1962). Silent Spring. Boston: Houghton Mifflin.

Foreman, D. (1990, abril). Only man's presence can save nature. Harper's Magazine, pp. $37-48$

Garlaschelli, E. y Petrosino, S. (2012). Lo stare degli uomini. Sul senso dell'abitare e sul suo dramma. Milano: Marietti 1820.

Haeckel, E. H. (1866). Allgemeine Anatomie der Organismen. Berlin: Druck und Verlag von Georg Reimer. seres vivientes: "Hay solo un pequeño paso de aquí a la Bio-ética [Bio-Ethik], es decir la asunción de deberes morales no solo hacia los seres humanos, sino también hacia todos los seres vivientes. De hecho, la Bio-ética no es solo un descubrimiento del presente. Como ejemplo particularmente impresionante del pasado podemos recordar la figura de San Francisco de
Heidegger, M. (1994). Construir, habitar, pensar. En: Heidegger, M. Conferencias y artículos. Barcelona: Ediciones del Serbal, pp. 127-142.

Heidegger, M. (2000). Vorträge und Aufsätze. En: Herrmann, F. W. von (ed.). Gesamtausgabe (Band 7). Frankfurt am Main: Vittorio Klostermann.

Heidegger, M. (2009). El Arte y el Espacio. Barcelona: Herder.

Jahr, F. (1927). Bio-Ethik. Eine Umschau über die ethischen Beziehungen des Menschen zu Tier und Pflanze. Kosmos. Handweiser für Naturfreunde, 24 (1), pp. 2-4.

Jonas, H. (1973). Technology and Responsibility: Reflections on the New Tasks of Ethics. Social Research, 40 (1), pp. 31-54.

Jonas, H. (1997). Técnica, medicina y ética. Sobre la práctica del principio responsabilidad. Barcelona: Paidós.

Leopold, A. (1949). A Sand Country Almanac and Sketches Here and There. New York: Oxford University Press.

Lima, A. I. (2000). Soleri. Architettura come ecologia umana. L'opera completa. Milano: Jaca Book.
Asís y su gran amor a los animales" (Jahr, 1927, p. 2).

2. Sin embargo, es interesante observar que, en este aspecto particular, el movimiento de la ecología profunda ha "traicionado" las intenciones originales de su fundador, Arne Næss, que nunca ha querido apoyar una imagen tan pesimista y radical del ser humano (Valera, 2015a, pp. 33-38).
Marcos, A. (2012a). Biología sistémica y filosofía de la naturaleza. Eikasia. Revista de Filosofía, 43, pp. 95-110.

Marcos, A. (2012b). Dependientes y racionales: la familia humana. Cuadernos de Bioética, 23 (1), pp. 83-95.

Marten, G. G. (2003). Human Ecology. Basics concepts for sustainable development. London: Earthscan.

Meadows, D. H., Meadows, D. L., Randers, J., Beherns III, W. W. (1972). Limits to Growth. New York: New American Library.

Nancy, J.-L. (1993). Le sense du monde. Paris: Galilée.

Natoli, S. (1999). Dizionario dei vizi e delle virtù. Milano: Feltrinelli.

Næss, A. (1973). The Shallow and the Deep, Long-Range Ecology Movement. A Summary. Inquiry: An Interdisciplinary Journal of Philosophy, 16 (1-4), pp. 95-100. https://doi. org/10.1080/00201747308601682

Næss, A. (1989). Ecology, Community and Lifestyle. Outline of an Ecosophy. Cambridge: Cambridge University Press. https://doi.org/10.1017/ CB09780511525599 
Ortega y Gasset, J. (1983). Historia como sistema. En: Ortega y Gasset, J. Obras completas (vol. 6). Madrid: Alianza.

Park, R. E. (1936). Human Ecology. The American Journal of Sociology, 42 (1), pp. 1-15. https://doi.org/10.1086/217327

Pesare, M. (2006). La sicurezza dei luoghi. Abitare come aver-cura. Quaderno di comunicazione, 6, pp. 83-97.

Petrosino, S. (2003). Babele. Architettura filosofía e linguaggio di un delirio. Genova: II Melangolo.

Petrosino, S. (2007). Capovolgimenti. La casa non è una tana, l'economia non è il business. Milano: Jaca Book.

Potter, V. R. (2002). Bioética, la ciencia de la supervivencia. Selecciones de Bioética, 1, pp. 121-139.

Scheler, M. (1960). Metafísica de la libertad. Buenos Aires: Nova.

Scheler, M. (1964). El puesto del hombre en el cosmos. Buenos Aires: Losada.
Sears, P. B. (1954). Human Ecology: A problem in synthesis. Science, 120 (3128) pp. 959-963. https://doi.org/10.1126/ science.120.3128.959

Snow, C. P. (2009). Las dos culturas. Buenos Aires: Nueva Visión Argentina.

Terry Rambo, A. (1983). Conceptual approaches to human ecology. Honolulu: East-West Environment Policy Institute.

Valera, L. (2011). Ecologia ed ecologie. Medicina nei secoli. Arte e scienza, 2 (3), pp. 1015-1044.

Valera, L. (2014a). Oikos e relazioni: I'abitare come cura dell'alterità. En: Pagliacci, D. (ed.). Differenze e relazioni. Cura dei legami Vol. II. Roma: Aracne.

Valera, L. (2014b). Un nuovo cancro per i pianeta? Natura ed essere umano nell'etica ambientale contemporanea. Teoria. Rivista di Filosofia, 24 (2), pp. 175-192.

Valera, L. (2015a). Introduzione. En: Næss, A. Introduzione all'ecologia. Pisa: ETS.
Valera, L. (2015b). Tecnologia ed ecologia. Dall'etica alla metafisica, dalla negazione del limite alla negazione dell'uomo. Pensamiento. Revista de Investigación e Información Filosófica, 71 (269), pp. 1453-1462. https://doi.org/10.14422/ pen.v71.i269.y2015.024

Valera, L. y Marcos, A. (2014). Desarrollo humano sostenible: una visión aristotélica. Isegoría, 51, pp. 671-690. https://doi. org/10.3989/isegoria.2014.051.07

Zimmermann, M. E. (1994). Contesting earth's future. Radical ecology and postmodernity. Berkeley: University of California Press.

\section{Otros recursos}

Certificat International d'Écologie Humaine (CIEH): Certificat International d'Écologie Humaine Convention, 1978. [En línea]. Disponible en https://web.univpau.fr/RECHERCHE/CIEH 